\title{
TOPOLOGICAL EQUIVALENCE OF FOLIATIONS OF HOMOGENEOUS SPACES
}

\author{
DAVE WITTE
}

\begin{abstract}
For $i=1,2$, let $\Gamma_{i}$ be a lattice in a connected Lie group $G_{i}$, and let $X_{i}$ be a connected Lie subgroup of $G_{i}$. The double cosets $\Gamma_{i} g X_{i}$ provide a foliation $\mathscr{F}_{i}$ of the homogeneous space $\Gamma_{i} \backslash G_{i}$. Assume that $X_{1}$ and $X_{2}$ are unimodular and that $\mathscr{F}_{1}$ has a dense leaf. If $G_{1}$ and $G_{2}$ are semisimple groups to which the Mostow Rigidity Theorem applies, or are simply connected nilpotent groups (or are certain more general solvable groups), we use an idea of D. Benardete to show that any topological equivalence of $\mathscr{F}_{1}$ and $\mathscr{F}_{2}$ must be the composition of two very elementary maps: an affine map and a map that takes each leaf to itself.
\end{abstract}

\section{INTRODUCTION}

Let $\Gamma$ be a lattice in a connected Lie group $G$. Any connected Lie subgroup $X$ of $G$ acts by translations on the homogeneous space $\Gamma \backslash G$; the orbits of this action are the leaves of a foliation of $\Gamma \backslash G$. We call this the foliation of $\Gamma \backslash G$ by cosets of $X$. (The leaves of this foliation are in natural one-to-one correspondence with the double cosets $\Gamma g X$.) Now suppose $\Gamma^{\prime}$ is a lattice in some other connected Lie group $G^{\prime}$, and that $X^{\prime}$ is a connected Lie subgroup of $G^{\prime}$. It is natural to ask whether the foliation corresponding to $X$ on $\Gamma \backslash G$ is topologically equivalent to the foliation corresponding to $X^{\prime}$ on $\Gamma^{\prime} \backslash G^{\prime}$.

(1.1) Main Theorem. For $i=1,2$, let $G_{i}$ be a connected Lie group, let $\Gamma_{i}$ be a lattice in $G_{i}$, and let $X_{i}$ be a connected Lie subgroup of $G_{i}$. Let $\mathscr{F}_{i}$ be the foliation of $\Gamma_{i} \backslash G_{i}$ by cosets of $X_{i}$. Assume

(a) $\mathscr{F}_{i}$ has a dense leaf; and

(b) $X_{i}$ is unimodular; and

(c) either

(i) $G_{1}$ and $G_{2}$ are nilpotent and simply connected; or

(ii) $G_{1}$ and $G_{2}$ are solvable and simply connected, and all the eigenvalues of every element of $\operatorname{Ad} G_{1}$ and of every element of $\operatorname{Ad} G_{2}$ are real; or

(iii) $G_{1}$ and $G_{2}$ are semisimple with trivial center and no compact factor, and $G_{1}$ has no normal subgroup $N$, isomorphic to $\operatorname{PSL}(2, \mathbf{R})$, such that $N \Gamma_{1}$ is a closed subgroup of $G_{1}$.

Received by the editors May 26, 1988.

1980 Mathematics Subject Classification (1985 Revision). Primary 22E40; Secondary 57R30. 
If $f: \Gamma_{1} \backslash G_{1} \rightarrow \Gamma_{2} \backslash G_{2}$ is a homeomorphism that maps each leaf of $\mathscr{F}_{1}$ onto a leaf of $\mathscr{F}_{2}$, then $f$ is the composition of two very elementary maps: an affine map and a homeomorphism of $\Gamma_{2} \backslash G_{2}$ that maps each leaf of $\mathscr{F}_{2}$ onto itself.

The proof of this theorem is mostly due to Benardete: he [3] proved a weaker version of this theorem that replaces assumption (b) by the much stronger assumption that $X_{i}$ is a one-parameter subgroup, and replaces the conclusion of the theorem by the weaker statement that some topological equivalence of $\mathscr{F}_{1}$ and $\mathscr{F}_{2}$ is an affine map. (Benardete omitted assumption (a) in cases (i) and (ii) of (c).) In this paper, we show that Benardete's method can be extended to higher-dimensional foliations and that the method has the power to show that every equivalence of the foliations is of an elementary form, as described in the theorem.

Benardete's method is exemplified by the following astonishingly simple proof for foliations of tori.

(1.2) Theorem (classical). Let $X$ and $Y$ be connected Lie subgroups of $\mathbf{R}^{n}$. Then the foliation of the $n$-torus $\mathbf{T}^{n}=\mathbf{R}^{n} / \mathbf{Z}^{n}$ by cosets of $X$ is topologically equivalent to the foliation of $\mathbf{T}^{n}$ by cosets of $Y$ if and only if there exists $\sigma \in$ $G L(n, \mathbf{Z})$ with $\sigma(X)=Y$.

Proof (cf. Benardete [3, Theorem 2.1]). Let $f$ be a homeomorphism of $\mathbf{T}^{n}$ that maps each leaf of the foliation by cosets of $X$ onto a leaf of the foliation by cosets of $Y$. By composing $f$ with a translation, we may assume $f\left(0+\mathbf{Z}^{n}\right)=$ $\left(0+\mathbf{Z}^{n}\right)$. Let $\tilde{f}$ be the lift of $f$ to a homeomorphism of $\mathbf{R}^{n}$, fixing 0 . Then $\left.\tilde{f}\right|_{\mathbf{Z}^{n}}$, the restriction of $f$ to $\mathbf{Z}^{n}$, is an automorphism of $\mathbf{Z}^{n}$. This means $\left.\tilde{f}\right|_{\mathbf{Z}^{n}} \in \mathrm{GL}(n, \mathbf{Z})$, so

$\left.(*) \tilde{f}\right|_{\mathbf{Z}^{n}}$ extends to an automorphism $\sigma$ of $\mathbf{R}^{n}$.

By composing $\tilde{f}$ with the inverse of $\sigma$, we may assume the restriction of $\tilde{f}$ to $\mathbf{Z}^{n}$ is the identity. Because

(**) $\mathbf{R}^{n} / \mathbf{Z}^{n}$ is compact,

this implies that $\tilde{f}$ moves points by a bounded amount (i.e., there is some constant $C$ with $d(v, \tilde{f}(v))<C$ for all $\left.v \in \mathbf{R}^{n}\right)$. Suppose $X \neq Y$, and assume, for definiteness, that $X \not \subset Y$. Now $X$ and $Y$ are connected Lie subgroups of $\mathbf{R}^{n}$; i.e., they are linear subspaces. Hence, it is obvious that

$(* * *)$ since $X \not \subset Y$, there are elements of $X$ that are arbitrarily far from $Y$.

We have seen that $\tilde{f}$ moves points a bounded amount; because there are elements of $X$ arbitrarily far from $Y$, this implies that $\tilde{f}(X) \not \subset Y$. We know, however, that $\tilde{f}$ maps $X$-orbits to $Y$-orbits, and fixes the origin, so we must have $\tilde{f}(X)=Y$. This is a contradiction.

(1.3) Corollary. Let $X$ and $Y$ be connected Lie subgroups of $\mathbf{R}^{n}$. Assume the foliation of $\mathbf{T}^{n}$ by cosets of $X$ has a dense leaf. Suppose $f$ is a homeomorphism of $\mathbf{T}^{n}$ that maps each leaf of the foliation of $\mathbf{T}^{n}$ by cosets of $X$ onto a leaf of 
the foliation by cosets of $Y$. Then there is

(1) an affine map $A: \mathbf{T}^{n} \rightarrow \mathbf{T}^{n}$ that takes each leaf of the foliation by cosets of $X$ onto a leaf of the foliation by cosets of $Y$, and

(2) a homeomorphism $\phi: \mathbf{T}^{n} \rightarrow \mathbf{T}^{n}$ that takes each leaf of the foliation by cosets of $Y$ onto itself, such that $f$ is the composition $\phi \circ A$.

Proof. Because of the theorem, we may assume $X=Y$. Let $v \in \mathbf{R}^{n}$. We have $\tilde{f}(v)=v+\delta(v)$ for some $\delta(v) \in \mathbf{R}^{n}$. Because $\tilde{f}$ maps $v+X$ to a leaf of the foliation by cosets of $X$, it must be true that $\delta$ is constant on $v+X$, modulo $X$; i.e., the function $\bar{\delta}: \mathbf{T}^{n} \rightarrow \mathbf{R}^{n} / X$ is constant on the leaves of the $X$-foliation. Because there is a dense leaf, this implies $\bar{\delta}$ is constant. By composing $\tilde{f}$ with a translation if necessary, we may assume $\tilde{f}$ fixes some point $v_{0}$; then $\bar{\delta}\left(v_{0}\right)=0$; therefore, $\bar{\delta}(v)=0$ for all $v$. This means that $\tilde{f}$ maps each leaf of the foliation by cosets of $X$ onto itself.

Benardete's proof is so simple that it is very powerful; the proof can be applied to any class of homogeneous spaces where the following three conditions are known to be true: $(*)$ every isomorphism of $\Gamma$ with $\Gamma^{\prime}$ extends to an isomorphism of $G$ with $G^{\prime},(* *) \Gamma \backslash G$ is compact, and $(* * *)$ if $X$ and $Y$ are two connected Lie subgroups of $G$, and $X \not \subset Y$, then $X$ diverges from $Y$.

These conditions sometimes fail, but they do hold if $G$ and $G^{\prime}$ are simply connected nilpotent groups or certain more general solvable groups (see $\S 5$ ).

Condition $(*)$ is true for a broad class of semisimple groups (this is the Mostow Rigidity Theorem 2.1). Condition (**) can fail for semisimple groups, but it doesn't matter: Benardete showed how to avoid the need for this hypothesis by working inside a compact subset of $\Gamma \backslash G$ (see steps 3 and 4 of the proof of Theorem 6.1). Condition $(* * *)$ can fail but, extending the work of Benardete for one-parameter subgroups, we show in $\S 4$ that if two unimodular subgroups do not diverge, then they differ by a compact subgroup (Theorem 4.9). In $\S 6$, we use this as a substitute for $(* * *)$ to prove the Main Theorem in the case of semisimple groups.

Here is the organization of the paper: $\S 1$, Introduction; $\S 2$, Preliminaries; $\S 3$, Preliminaries on real algebraic groups; $\S 4$, Divergence; $\S 5$, Topological rigidity of homogeneous foliations: the solvable case; $\S 6$, Topological rigidity of homogeneous foliations: the semisimple case; $\S 7$, Complements; $\S 8$, Foliations of double-coset spaces.

Remark. For accuracy, we should point out that our results are not quite a generalization of Benardete's results, because Benardete assumed the existence of a topological equivalence that preserves the orientation on each leaf and was then able to conclude the existence of an affine map that preserves the orientation on each leaf. It is easier to ignore this restriction (which is what we have done), but, in the one-dimensional case considered by Benardete, it would not be difficult to transfer our results to the orientation-preserving setting. 
Acknowledgments. This work was supported by a postdoctoral fellowship from the National Science Foundation. It is based on Benardete's work [3]. Comments of A. Katok, I. M. Isaacs, A. Nevo, and M. Ratner were helpful in the preparation of this paper.

\section{Preliminaries}

Varadarajan's book [17] is a good reference for the basic concepts in the study of Lie groups and Lie algebras; Raghunathan's book [14] is a good reference for material on lattice subgroups. Additional material on both of these topics appears in Zimmer's book [18].

Definition. A map between homogeneous spaces is said to be an affine map if it is the composition of a group homomorphism and a translation. More precisely, suppose $\Gamma_{1}$ and $\Gamma_{2}$ are lattices in Lie groups $G_{1}$ and $G_{2}$. A continuous map $f: \Gamma_{1} \backslash G_{1} \rightarrow \Gamma_{2} \backslash G_{2}$ is an affine map if there are a continuous homomorphism $\sigma: G_{1} \rightarrow G_{2}$ with $\sigma\left(\Gamma_{1}\right) \subset \Gamma_{2}$ and an element $g$ of $G_{2}$, such that $f\left(\Gamma_{1} x\right)=$ $\Gamma_{2} \cdot \sigma(x) \cdot g$, for every $x \in G_{1}$.

Definition. Let $\mathscr{Y}$ be the Lie algebra of a Lie group $Y$, of dimension $d$. Then $\bigwedge^{d} \mathscr{Y}$, the $d$ th exterior power of $\mathscr{Y}$, is one-dimensional, and $Y$ acts on $\bigwedge^{d} \mathscr{Y}$ by an exterior power of the adjoint representation. We say $Y$ is unimodular if the representation of $Y$ on $\bigwedge^{d} \mathscr{Y}$ is trivial. (This is equivalent to the more usual definition that right and left Haar measure agree.)

The proof of Theorem 6.1 is based on the following deep theorem. This theorem was first proved, by D. Mostow, only for cocompact lattices; it was extended to noncocompact lattices by G. Prasad and G. A. Margulis.

(2.1) Theorem (Moscow Rigidity Theorem, Mostow [12, Theorem 24.1, p. 187], Prasad [13], Margulis [18, Theorem 5.1.1, p. 85]). For $i=1,2$, let $G_{i}$ be a connected, semisimple Lie group with trivial center and no compact factor; let $\Gamma_{i}$ be a lattice in $G_{i}$. Assume there is no closed, normal subgroup $N$ of $G_{1}$ such that $N$ is isomorphic to PSL $(2, \mathbf{R})$, and $N \Gamma_{1}$ is closed. If $\sigma: \Gamma_{1} \rightarrow \Gamma_{2}$ is any group isomorphism, then $\sigma$ extends to an isomorphism of $G_{1}$ with $G_{2}$.

The proof of Theorem 5.1 will use an analogous result for solvable groups. In the special case of nilpotent groups, the appropriate theorem was proved by Malcev (see [14, Theorem II.2.11, p. 33]). Some restrictions are necessary if one wishes to extend Malcev's theorem to a more general class of solvable groups (see $[3, \S 10 \mathrm{f}]$ for a counterexample). That is why we make a restriction on the eigenvalues of Ad $g$ for $g \in G$.

(2.2) Theorem (Saito, [16, Theorem 5], or [5, Proposition 2.1]). For $i=1,2$ let $G_{i}$ be a simply connected, solvable Lie group; let $\Gamma_{i}$ be a lattice in $G_{i}$. Assume, for every $g \in G$, that every eigenvalue of $\operatorname{Ad} g$ is real. If $\sigma: \Gamma_{1} \rightarrow \Gamma_{2}$ is any group isomorphism, then $\sigma$ extends to an isomorphism of $G_{1}$ with $G_{2}$.

(2.3) Lemma [14, Theorem 3.1, p. 43]. If $\Gamma$ is a lattice in a connected, solvable Lie group $G$, then $\Gamma \backslash G$ is compact. 
(2.4) Lemma [14, $\S 1.8$ of Preliminaries, p. 6]. Let $G$ be a connected Lie group. Then $G$ has maximal compact subgroups. If $K$ is any maximal compact subgroup of $G$, then the conjugates of $K$ are all the maximal compact subgroups of $G$ : every compact subgroup of $G$ is contained in some conjugate of $K$.

(2.5) Lemma. Let $A, B$, and $C$ be subgroups of a group $G$. If $A \subset C$, then $A \cdot(B \cap C)=(A B) \cap C$.

(2.6) Lemma [17, Exercise 3.36, p. 254]. Let $G$ be a simply connected, solvable Lie group. Then $G$ has no nontrivial, compact subgroups.

(2.7) Lemma (Poincaré Recurrence Theorem [18, Lemma 9.1.5]). Let $a: S \rightarrow S$ be a measure-space isomorphism preserving the finite measure $\mu$. Let $K \subset S$ with $\mu(K)>0$. Then, for almost all $x \in K$, we have $a^{n}(x) \in K$ for infinitely many positive values of $n$ and for infinitely many negative values of $n$.

(2.8) Lemma (weak mixing). Let $X$ be a connected Lie subgroup of a connected, semisimple Lie group $G$ with trivial center and no compact factor; let $\Gamma$ be a lattice in $G$; and let $\mathscr{F}$ be the foliation of $\Gamma \backslash G$ by cosets of $X$. Let $K$ be a closed subgroup of a compact Lie group $E$. Assume $\mathscr{F}$ has a dense leaf, and suppose there is a continuous map $\delta: \Gamma \backslash G \rightarrow E / K$ such that, for each $x \in X$, there is some $e=e_{x} \in E$ with $e \cdot \delta(\Gamma g x)=\delta(\Gamma g)$ for all $g \in G$. Then

(a) $\delta$ is constant, and

(b) $\left\{e_{x} \mid x \in X\right\}$ is contained in a conjugate of $K$.

Proof. Conclusion (b) is a consequence of (a), so we will prove only (a). Because $\mathscr{F}$ has a dense leaf, it is easy to see that there must be some element $x$ of $X$ for which there is no closed, proper, normal subgroup $N$ of $G$ such that $\Gamma$ projects to a discrete subgroup of $G / N$ and such that the subgroup generated by $x$ projects to a precompact subgroup of $G / N$. Hence, the Moore Ergodicity Theorem [11, Theorem 3] implies that the action of $x$ by translation on $\Gamma \backslash G$ has a dense orbit. In fact, Moore's theorem implies that the action is weak mixing, so the action of $x$ by translation on $\Gamma \backslash G$ has no nonconstant eigenfunctions [4, Theorem 1.7.2, p. 29].

Let $D$ be the closure of the subgroup generated by $e=e_{x}$ in $E$. The map $\delta: \Gamma \backslash G \rightarrow E / K$ induces a map $\bar{\delta}: \Gamma \backslash G \rightarrow D \backslash E / K$. Because $e \cdot \delta(\Gamma g x)=$ $\delta(\Gamma g)$, we have $\bar{\delta}(\Gamma g x)=\bar{\delta}(\Gamma g)$ for all $g \in G$. Because $x$ has a dense orbit on $\Gamma \backslash G$, this implies that $\bar{\delta}$ is constant. Hence the range of $\delta$ lies in a single $D$-orbit on $E / K$; by restricting our attention to this $D$-orbit, we may assume $E=D$; in particular, we may assume that $E$ is abelian.

Let $\chi$ be any character of the abelian group $E / K$. Then

$$
\chi(\delta(g x))=\chi(e \cdot \delta(g))=\chi(e) \cdot \chi(\delta(g))
$$

so the composition $\chi \circ \delta$ is an eigenfunction for $x$. The conclusion of the first paragraph of the proof then asserts that $\chi \circ \delta$ is constant. Because this is true for all characters $\chi$ of $E / K$, we conclude that $\delta$ is constant. 


\section{Preliminaries on real algebraic groups}

In this section, we will establish some fundamental properties of the elliptic part and the nonelliptic part of a connected Lie subgroup of $\operatorname{GL}(n, \mathbf{R})$ (see Definition 4.8). To do so, we will need some basic properties of real algebraic groups. (This section assumes more algebraic background than the rest of the paper.) The books of Humphreys [9] and Hochschild [8] provide good introductions to the theory of algebraic groups. The books of Humphreys [9, §34], Raghunathan [14, $\S 2$ of Preliminaries], and Zimmer [18, §3.1] contain useful information specifically on algebraic groups over $\mathbf{R}$.

Definition (adapted from [14, $\S 2$ of Preliminaries, pp. 7-8]). Recall that $\mathrm{GL}(n, \mathbf{R})$ acquires the structure of a real algebraic variety through the embedding $\operatorname{GL}(n, \mathbf{R}) \hookrightarrow \mathbf{R}^{n^{2}+1}$ defined by $g \mapsto\left(g_{i j},(\operatorname{det} g)^{-1}\right)$.

A function $f$ on this variety is regular if there is a polynomial $p$ in $n^{2}+1$ variables such that $f(g)=p\left(g_{i j},(\operatorname{det} g)^{-1}\right)$; we usually refer to regular functions simply as polynomials.

A subgroup $G$ of $\operatorname{GL}(n, \mathbf{R})$ is Zariski closed if $G$ is an algebraic subvariety, i.e., if $G$ is the set of zeros of some collection of polynomial functions on $\mathrm{GL}(n, \mathbf{R})$. (In the literature, such a group $G$ is often called an algebraic group over $\mathbf{R}$.)

For any subgroup $X$ of $\operatorname{GL}(n, \mathbf{R})$, the Zariski closure of $X$ is the (unique) smallest Zariski-closed subgroup containing $X$.

Remark. It is easy to embed $\operatorname{GL}(n, \mathbf{R})$ in $\operatorname{SL}(n+1, \mathbf{R})$, so one sees that every Zariski-closed subgroup of $\mathrm{GL}(n, \mathbf{R})$ can be realized as a Zariski-closed subgroup of some special linear group. Given a Zariski-closed subgroup $G \subset$ $\mathrm{GL}(n, \mathbf{R})$, it simplifies matters slightly to assume that $G$ is contained in $\mathrm{SL}(n, \mathbf{R})$, because then a regular function on $G$ is simply a polynomial in the matrix entries of $g$.

Definition. A connected Lie subgroup $G$ of $\operatorname{GL}(n, \mathbf{R})$ is a connected real algebraic group if there is a Zariski-closed subgroup $G^{*}$ of $\operatorname{GL}(n, \mathbf{R})$ such that $G$ is the identity component (in the Hausdorff topology) of $G^{*}$ (i.e., if $G=\left(G^{*}\right)^{0}$ ).

Definition [17, p. 151]. Let $x \in \operatorname{GL}(n, \mathbf{R})$. We say $x$ is semisimple if $x$ is diagonalizable over $\mathbf{C}$.

Definition [6, §IX.7, p. 430]. Let $x \in \mathrm{GL}(n, \mathbf{R})$. We say that $x$ is hyperbolic if $x$ is semisimple and all of its eigenvalues are real and positive. We say that $x$ is unipotent if 1 is the only eigenvalue of $x$. We say that $x$ is elliptic if $x$ is semisimple and all the eigenvalues of $x$ have absolute value one.

Remark [6, Theorem IX.7.2(i), p. 431]. A matrix $x \in \mathrm{GL}(n, \mathbf{R})$ is elliptic if and only if it belongs to some compact subgroup of $\operatorname{GL}(n, \mathbf{R})$. 
(3.1) Lemma [6, Lemma IX.7.1, p. 430]. Let $g \in G L(n, \mathbf{R})$. Then $g$ has a unique decomposition $g=$ hue, where $h$ is hyperbolic, $u$ is unipotent, $e$ is elliptic, and each of $g, h, u$, and e commutes with the other three.

(3.2) Lemma (cf. proof of [9, Theorem 15.3, p. 99]). Let $g \in G L(n, \mathbf{R})$ and suppose $g=h u e$, where $h$ is hyperbolic, $u$ is unipotent, $e$ is elliptic, and each of $g, h, u$, and $e$ commutes with the other three. If $G$ is a Zariski-closed subgroup of $G L(n, \mathbf{R})$ and if $G$ contains $g$, then $G$ contains $h, u$, and $e$.

(3.3) Lemma. Let $X$ be a one-parameter subgroup of a Zariski-closed subgroup $G$ of $G L(n, \mathbf{R})$, and let $Y$ be the nonelliptic part of $X$. Then $Y$ is contained in $G$.

Proof. Let $\rho: \mathbf{R} \rightarrow G$ with $\rho(\mathbf{R})=X$; write $\rho(t)=\rho^{+}(t) \cdot \rho^{0}(t)$ as in Definition 4.3. Fix $t \in \mathbf{R}$; it suffices to show that $\rho^{+}(t) \in G$. Let $g=\rho(t)$, and write $g=h u e$ as in Lemma 3.2. Then it is not hard to see that $\rho^{+}(t)=h u$, so $\rho^{+}(t)$ belongs to $G$, as desired.

(3.4) Lemma [8, Proposition VIII.3.1, p. 112]. Let $Y^{*}$ be the Zariski closure of a connected Lie subgroup $Y$ of $G L(n, \mathbf{R})$. Then $\left[Y^{*}, Y^{*}\right]^{0} \subset Y$.

(3.5) Lemma. Let $X$ be a connected Lie subgroup of $G L(n, \mathbf{R})$. Then the Zariski closure of $X$ normalizes $X$.

Proof. This follows from Lemma 3.4. For a more elementary proof, see the proof of [18, Theorem 3.2.5(ii), p. 42)].

(3.6) Lemma. Let $G$ be a connected real algebraic subgroup of $G L(n, \mathbf{R})$. Then $G$ has connected real algebraic subgroups $L, R$, and $E$ such that

(1) $G=L R E$;

(2) $L$ is semisimple with no compact factor,

(3) $R$ is solvable and simply connected;

(4) $E$ is compact;

(5) $R \triangleleft G$;

(6) $[L, E]=e$;

(7) $L \cap R E, R \cap E L$, and $E \cap L R$ are finite.

Proof. Let $U$ be the unipotent radical of $G$. (Thus, $U$ is a simply connected, nilpotent group.) It is well known [8, Theorem VIII.4.3, p. 117] that there is a reductive, connected, real algebraic subgroup $P$ of $G$ with $G=P \ltimes U$. By the structure of reductive algebraic groups [14, $\S 2.5$ of Preliminaries, p. 11], there are normal, connected, real algebraic subgroups $Q$ and $T$ of $P$ with $P=Q T$, such that $Q \cap T$ is finite, $Q$ is semisimple, $T$ is a torus, and $[Q, T]=e$.

We may write $T=A^{\prime} \times T^{\prime}$, where $A^{\prime}$ is an $\mathbf{R}$-split torus and $T^{\prime}$ is an anisotropic torus [9, Theorem $3.43 \mathrm{~b}$, p. 219]. Let $L^{\prime}$ be the product of all the noncompact simple factors of $Q$, and let $E^{\prime}$ be the maximal connected, compact, normal subgroup of $Q$. Then one can verify that $L^{\prime}, E^{\prime}, A^{\prime}$, and $T^{\prime}$ are normal, connected, real algebraic subgroups of $P$ such that

(1) $P=L^{\prime} E^{\prime} A^{\prime} T^{\prime}$; 
(2) $L^{\prime} E^{\prime}$ is semisimple, and $L^{\prime}$ has no compact factor;

(3) $E^{\prime}$ and $T^{\prime}$ are compact;

(4) $T^{\prime}$ and $A^{\prime}$ are abelian, and $A^{\prime}$ is simply connected;

(5) each of the subgroups $L^{\prime}, E^{\prime}, A^{\prime}$, and $T^{\prime}$ centralizes the other three and has finite intersection with the product of the other three;

(6) each of the subgroups $L^{\prime}, E^{\prime}, A^{\prime}$, and $T^{\prime}$ is a normal subgroup of $P$;

Let $L=L^{\prime}, R=A^{\prime} U$, and $E=E^{\prime} T^{\prime}$. The required properties of $L, R$, and $E$ are not difficult to verify from the properties of $L^{\prime}, E^{\prime}, A^{\prime}$, and $T^{\prime}$.

(3.7) Lemma. Let $X$ be a connected Lie subgroup of $G L(n, \mathbf{R})$. Let $X^{*}$ be the identity component of the Zeriski closure of $X$, and let $Y$ be the nonelliptic part of $X$. Write $X^{*}=L R E$ as in Lemma 3.6. Then $Y=X E \cap L R$. In particular, $L$ is contained in $Y$; and $\operatorname{rad} Y$ is contained in $R$, so $\operatorname{rad} Y$ is closed and simply connected.

Proof. We break the argument into several steps.

Step 1. We have $Y \subset X E$. Let $K$ be a maximal compact subgroup of $L E$; then $K R / R$ is a maximal compact subgroup of $X^{*} / R$. Because $R$ has no nontrivial, compact subgroups (see Lemma 2.6), this implies that $K$ is a maximal compact subgroup of $X^{*}$. So Lemma 2.4 implies that any compact subgroup of $X^{*}$ is contained in a conjugate of $K$. Because Lemma 3.4 implies $L \subset X$, we have $K \subset L E \subset X E$. Then, since Lemma 3.4 implies $X E \triangleleft X^{*}$, every compact subgroup of $X^{*}$ is contained in $X E$.

Let $A$ be a one-parameter subgroup of $X$; let $Y_{A}$ be the nonelliptic part of $A$, and let $E_{A}$ be the elliptic part of $A$. Then $E_{A}$ is a compact subgroup of $X^{*}$, so the conclusion of the preceding paragraph asserts that $E_{A} \subset X E$. Because $Y_{A} \subset A \cdot E_{A}$ and $A \subset X$, this implies that $Y_{A} \subset X \cdot X E=X E$. Thus, $X E$ contains the nonelliptic part of an arbitrary one-parameter subgroup of $X$; hence, $Y \subset X E$.

Step 2. We have $Y \subset L R$. Any compact, connected, real algebraic group (such as $X^{*} /(L R)$ ) has no hyperbolic or unipotent elements. Hence, every hyperbolic element and every unipotent element of $X^{*}$ belong to $L R$; hence $Y \subset L R$.

Step 3. $X E \cap L R \subset Y$. We know $L E \cap R$ is finite, so Lemma 2.6 implies $L E \cap R=e$; therefore, $X^{*}=(L E) \ltimes R$. Lemma 3.4 implies $L \subset X$; hence $L E \subset X E$. Thus, $X E=L E \ltimes(X E \cap R)$, and $X E \cap L R=L \ltimes(X E \cap R)$. (In particular, because $X E$ is connected, we know that $X E \cap R$ is connected.) Now $L \subset X$, and $L$ is generated by one-parameter, unipotent subgroups, so $L \subset Y$. So we need only show $X E \cap R \subset Y$.

Let $N=\left[X^{*}, R\right]$. Then Lemma 3.4 implies $N \subset X$. Because no oneparameter subgroup of $R$ has an elliptic part, we also have $N \subset Y$. Let $\bar{r}(t)$ be a one-parameter subgroup of $(R \cap X E) / N$. By the definition of $N$, we know that $R / N$ centralizes $E N / N$, so, by the choice of $\vec{r}(t)$, there is a one-parameter subgroup $\bar{e}(t)$ of $E / N$ such that $\bar{r}(t) \cdot \bar{e}(t)$ is a one-parameter subgroup of $X / N$; let $x(t)$ be a one-parameter subgroup of $X$ with $x(t) \cdot N=\bar{r}(t) \cdot \bar{e}(t)$. 
Let $y(t)$ be the nonelliptic part of $x(t)$. Then $y(t) \cdot N$ is the nonelliptic part of $x(t) \cdot N=\bar{r}(t) \cdot \bar{e}(t)$, so $y(t) \cdot N=\bar{r}(t)$. Hence, $\bar{r}(t) \in Y / N$.

We have shown that $Y / N$ contains every one-parameter subgroup of $(R \cap X E) / N$. Because $R \cap X E$ is connected, this implies that $Y$ contains $R \cap X E$, as desired.

Step 4. We have $L \subset Y$. Lemma 3.4 implies $L \subset X$. So step 3 implies $L \subset Y$.

Step 5. We have $\operatorname{rad} Y \subset R$, so $\operatorname{rad} Y$ is closed and simply connected. It is obvious that $R$ and $Y$ normalize $R \cdot \operatorname{rad} Y$. Then, from step 4, we see that $L R$ normalizes $R \cdot \operatorname{rad} Y$. Because $R=\operatorname{rad}(L R)$, and $R \cdot \operatorname{rad} Y$ is solvable, this implies $R \cdot \operatorname{rad} Y \subset R$; hence, $\operatorname{rad} Y \subset R$. Every connected Lie subgroup of a simply connected solvable group is closed and simply connected [17, Theorem 3.18 .12$, p. 243], so this implies $\operatorname{rad} Y$ is closed and simply connected.

(3.8) Lemma. Let $Y$ be a connected Lie subgroup of $G L(n, \mathbf{R})$, and let $L$ be a Levi subgroup of $\bar{Y}$. Then

(i) $L \subset Y$;

(ii) $L \cap \operatorname{rad} Y$ is finite;

(iii) if $\operatorname{rad} Y$ is simply connected, then $L \cap \operatorname{rad} Y=e$; and

(iv) if $\operatorname{rad} Y$ is closed, then $Y$ is closed.

Proof. (i) Let $Y^{*}$ be the Zariski closure of $Y$. Then Lemma 3.4 implies $\left[Y^{*}, Y^{*}\right]^{0} \subset Y$. Because $\bar{Y} \subset Y^{*}$, this implies $[\bar{Y}, \bar{Y}] \subset Y$. In particular, $L \subset Y$.

(ii) Let $L^{*}$ be the Zariski closure of $L$, and let $R^{*}$ be the Zariski closure of $\operatorname{rad} Y$. Then $L^{*} \cap R^{*}$ is a Zariski closed, solvable normal subgroup of $L^{*}$. Because $L^{*}$ is semisimple, then $L^{*} \cap R^{*}$ must be finite. Because $L \subset L^{*}$ and $\operatorname{rad} Y \subset R^{*}$, this implies $L \cap \operatorname{rad} Y$ is finite.

(iii) If $\operatorname{rad} Y$ is simply connected, then Lemma 2.6 implies that $\operatorname{rad} Y$ has no nontrivial finite subgroups. Then, from (ii), we conclude that $L \cap \operatorname{rad} Y=e$.

(iv) We know $\bar{Y}=L \cdot \operatorname{rad} \bar{Y}$, and (ii) asserts that $L \cap \operatorname{rad} \bar{Y}$ is finite. Hence the map $\eta: \operatorname{rad} Y \rightarrow \bar{Y} / L: y \mapsto y L$ is a proper map. By hypothesis, $\operatorname{rad} Y$ is a closed subset of $\operatorname{rad} \bar{Y}$. Because $\eta$ is a proper map, this implies $\eta(\operatorname{rad} Y)$ is a closed subset of $\bar{Y} / L$; i.e., $(\operatorname{rad} Y) \cdot L$ is a closed subset of $\bar{Y}$. Because (i) implies that $(\operatorname{rad} Y) \cdot L=Y$, this means that $Y$ is closed.

(3.9) Proposition. Let $X$ be a connected Lie subgroup of $G L(n, \mathbf{R})$, with nonelliptic part $Y$. Then:

(i) $X$ and $Y$ normalize each other;

(ii) $Y$ is a closed, connected subgroup of $G L(n, \mathbf{R})$;

(iii) if $N$ is a connected, closed, normal Lie subgroup of $Y$, such that either $N$ or $Y / N$ is compact, then either $N$ is trivial or $N=Y$, respectively.

Proof. (i) Because $X$ is a connected Lie subgroup, Lemma 3.5 asserts that $X^{*}$ normalizes $X$; this implies that $Y$ normalizes $X$. Because $Y$ is defined from $X$, it is obvious that $X$ normalizes $Y$. (Indeed, all of $X^{*}$ normalizes $Y$.) 
The proof of (ii), (iii), and (iv) will rely on Lemma 3.7. Let $X^{*}$ be the identity component of the Zariski closure of $X$. Write $X^{*}=L R E$ as in Lemma 3.6.

(ii) From the definition of $Y$ (see 4.8), it is immediate that $Y$ is generated by one-parameter subgroups. Hence, $Y$ is connected. Lemma 3.7 asserts $\operatorname{rad} Y$ is closed, so Lemma 3.8(iv) implies $Y$ is closed.

(iii) Suppose $N$ is compact. Because $L \cap \operatorname{rad} Y$ is finite (see Lemma 3.8(ii)), this implies that $L \cap(N \cdot \operatorname{rad} Y)$ is compact. We also know that $L$ normalizes $N \cdot \operatorname{rad} Y$. Because $L$ has no connected, compact normal subgroups, then $L \cap(N \cdot \operatorname{rad} Y)$ is finite. Because $L \cdot \operatorname{rad} Y=Y$, and $N$ is connected, this implies $N \subset \operatorname{rad} Y$. Hence (see Lemma 3.7), $N \subset R$. So Lemma 2.6 implies $N=e$.

Suppose $Y / N$ is compact. Because $L \subset Y$ (see Lemma 3.7), and $L$ has no compact factors, this implies $L \subset N$. Because $Y=L \cdot \operatorname{rad} Y$, then $Y / N \cong$ $\operatorname{rad} Y /(N \cap \operatorname{rad} Y)$; so $Y / N$ is solvable. Also, $N=L \cdot(N \cap \operatorname{rad} Y)$. Because $N$ is connected and $L \cap \operatorname{rad} Y=e$ (see Lemma 3.8(iii)), this implies $N \cap \operatorname{rad} Y$ is connected. Because $\operatorname{rad} Y$ is simply connected, then $\operatorname{rad} Y /(N \cap \operatorname{rad} Y)$ is simply connected. Therefore, $Y / N$ is a compact, simply connected, solvable group. Hence Lemma 2.6 implies $Y / N=e$.

(3.10) Proposition. Let $X$ be a connected Lie subgroup of $G L(n, \mathbf{R})$, with nonelliptic part $Y$. Then there is a compact, connected subgroup $E$ of $G L(n, \mathbf{R})$, such that:

(a) $X E=Y E=\overline{X Y}$;

(b) $E \cap Y$ is finite;

(c) $E$ normalizes both $X$ and $Y$;

(d) $[E, Y] \subset X \cap Y$;

(e) $E \cap X Y$ is dense in $E$.

Proof. We will rely on Lemma 3.7. Let $X^{*}$ be the identity component of the Zariski closure of $X$. Write $X^{*}=L R E$ as in Lemma 3.6.

(b) Because $E \cap L R$ is finite, and $Y \subset L R$, it is obvious that $E \cap Y$ is finite.

(c) Because $E \subset X^{*}$, we know that $E$ normalizes both $X$ and $Y$.

(d) Lemma 3.4 asserts $\left[X^{*}, X^{*}\right] \subset X$. Because $E, Y \subset X^{*}$, this implies $[E, Y] \subset X$. Because $E$ normalizes $Y$, we have $[E, Y] \subset Y$.

(e) By Lemma 3.7 and Lemma 2.5, we have

$$
X Y=X \cdot(X E \cap L R)=X E \cap X L R .
$$

Therefore, $E \cap X Y=E \cap X L R$, so it suffices to show $E \cap X L R$ is dense in $E$.

Let $F$ be the identity component of the Zariski closure of $E \cap X L R$. Then $L R F$ is a connected real algebraic group, contains $X$, and is contained in $L R E$. Because $L R E$ is the identity component of the Zariski closure of $X$, this implies $L R F=L R E$. Hence $F=E$, so $E \cap X L R$ is Zariski dense in $E$. Because every compact subgroup of $\operatorname{GL}(n, \mathbf{R})$ is Zariski closed [2, Theorem 16, p. 116], this implies that $E \cap X L R$ is dense in $E$, as desired. 
(a) Lemma 3.7 asserts $Y=X E \cap L R E$. Because $X$ and $E$ are contained in $X^{*}=L R E$, we have

$$
Y E=(X E \cap L R E) \cdot E=(X E) \cdot E=X E .
$$

Obviously, $Y \subset \overline{X Y}$; and (e) implies $E \subset \overline{X Y}$. We know that $Y$ is closed, and $E$ is compact, so it is obvious that $Y E$ is closed. Hence $Y E=\overline{X Y}$.

\section{DivergenCE}

In this section, we will give a short proof of Benardete's main result on divergence of one-parameter subgroups (Theorem 4.7) and then show how this proof generalizes to a result on divergence of unimodular subgroups (Theorem 4.9).

Definition. Let $X$ and $Y$ be subsets of a Lie group $G$. We say that $Y$ diverges from $X$ if there is no compact subset $K$ of $G$ with $Y \subset X \cdot K$.

Remark. If $X$ and $Y$ are subgroups, then $Y \subset X \cdot K$ iff $Y \subset K^{-1} \cdot X$, so, for subgroups, it makes no difference in this definition whether $K$ is multiplied on the right of $X$ or on the left.

(4.1) Example. Let $G$ be a simply connected, abelian Lie group (so $G \cong \mathbf{R}^{n}$ ). If $X$ and $Y$ are connected Lie subgroups of $G$, and if $X$ does not diverge from $Y$, then $X \subset Y$. If, in addition, $Y$ does not diverge from $X$, we conclude that $X=Y$.

Definition. A subset $X$ of $\mathbf{R}$ is doubly unbounded if the infimum of $X$ is $-\infty$ and the supremum of $X$ is $+\infty$. Let $A$ be a one-parameter subgroup of a Lie group; let $\rho: \mathbf{R} \rightarrow A$ be a surjective homomorphism. A subset $X$ of $A$ is doubly unbounded if $\rho^{-1}(X)$ is doubly unbounded in $\mathbf{R}$.

D. Benardete [3] adopted an a priori stronger notion of divergence for his work on one-parameter subgroups: in our terminology, he assumed that every doubly unbounded subset diverges. (We will see in steps 3 and 4 of the proof of Theorem 6.1 that the stronger notion is important for the study of foliations of noncompact homogeneous spaces of finite volume.) Corollary 4.13 will show, for one-parameter subgroups, that this apparently stronger property follows automatically from our definition of divergence.

(4.2) Lemma (Real Canonical Form, cf. [7, pp. 126-130]). Let $M$ be any $n \times n$ real matrix. Then $M$ has a unique decomposition $M=D+N+E$, where $D$ is diagonalizable, and all of its eigenvalues are real, $N$ is nilpotent, $E$ is conjugate to a skew-symmetric matrix, and such that each of $M, D, N$, and $E$ commutes with the other three.

(4.3) Definition. Let $X$ be a one-parameter subgroup of $\operatorname{GL}(n, \mathbf{R})$. Then there is a homomorphism $\rho: \mathbf{R} \rightarrow \mathrm{GL}(n, \mathbf{R})$ with $\rho(\mathbf{R})=X$. Being a homomorphism from $\mathbf{R}$ to $\mathrm{GL}(n, \mathbf{R}), \rho$ must be of the form $\rho(t)=e^{M t}$ for 
some real $n \times n$ matrix $M$; write $M=D+N+E$ as in Lemma 4.2. Then we may write $\rho(t)=\rho^{+}(t) \cdot \rho^{0}(t)$, where $\rho^{+}(t)=e^{(D+N) t}$ and $\rho^{0}(t)=e^{E t}$. The one-parameter subgroup $\rho^{+}(\mathbf{R})$ is the nonelliptic part of $X$; the closure of $\rho^{0}(\mathbf{R})$ is the elliptic part of $X$. The nonelliptic part is a closed, simply connected, one-parameter subgroup of $\operatorname{GL}(n, \mathbf{R})$; the elliptic part is a compact, connected, abelian subgroup, but may be higher-dimensional.

(4.4) Lemma. Let $X_{1}$ and $X_{2}$ be one-parameter subgroups of $G L(n, \mathbf{R})$. If $X_{1}$ and $X_{2}$ have the same nonelliptic part, then they do not diverge from each other.

Proof. Let $E_{i}$ be the elliptic part of $X_{i}$; let $Y$ be the nonelliptic part of $X_{1}$ and $X_{2}$. Then $X_{1} \subset Y E_{1} \subset X_{2} E_{2} E_{1}$. Because $E_{2} E_{1}$ is compact, this implies that $X_{1}$ does not diverge from $X_{2}$. Similarly, $X_{2}$ does not diverge from $X_{1}$.

Definition. Suppose $f$ is a real-valued function on the real line. For convenience, let us say that $f$ is a polynomial of exponentials if there are a natural number $n \geq 0$, real numbers $\lambda_{1}, \ldots, \lambda_{n}$, and a real polynomial $P\left(x_{0}, \ldots, x_{n}\right)$ in $n+1$ variables such that, for $t \in \mathbf{R}$, we have $f(t)=P\left(t, e^{\lambda_{1} t}, e^{\lambda_{2} t}, \ldots, e^{\lambda_{n} t}\right)$.

(4.5) Lemma (a trivial exercise). Let $f$ be a polynomial of exponentials. If $f$ is not constant, then either $\lim _{t \rightarrow \infty}|f(t)|=\infty$, or $\lim _{t \rightarrow-\infty}|f(t)|=\infty$.

(4.6) Lemma. Let $\rho: \mathbf{R} \rightarrow G L(n, \mathbf{R})$ be a homomorphism, and assume $\rho(\mathbf{R})$ has no elliptic part. Then, as a function of $t$, each matrix entry of $\rho(t)$ is a polynomial of exponentials.

Proof. The homomorphism $\rho$ must be of the form $\rho(t)=e^{M t}$ for some real $n \times n$ matrix $M$. The assumption that $\rho$ has no elliptic part means that, in a suitable basis, $M$ is of the form $M=D+N$, where $D$ is diagonal, $N$ is nilpotent, and $D$ commutes with $N$. Since $D$ and $N$ commute, we have $e^{(D+N) t}=e^{D t} e^{N t}$. Because $N$ is nilpotent, $e^{N t}$ is just a polynomial in $t$ (with matrix coefficients); because $D$ is diagonal, $e^{D t}$ is just a diagonal matrix of exponentials of $t$. Thus, each matrix entry of $e^{N t}$ and $e^{D t}$ is a polynomial of exponentials; multiplying these matrices of polynomials of exponentials results in a matrix (and this matrix is $\rho(t)$ ) whose entries are polynomials of exponentials.

(4.7) Theorem (Benardete [3, Theorem 3 (of $\S 4$ )]). Let $X_{1}$ and $X_{2}$ be oneparameter subgroups of $G=G L(n, \mathbf{R})$. If $X_{1}$ and $X_{2}$ do not diverge from each other, then they have the same nonelliptic part; they can differ only in the elliptic part.

Proof. Let $E_{i}$ be the elliptic part of $X_{i}$, and let $Y_{i}$ be the nonelliptic part of $X_{i}$. Note that, of course, $Y_{1}$ and $Y_{2}$ have no elliptic part. Because $X_{1}$ and $X_{2}$ do not diverge from each other, $Y_{1}$ and $Y_{2}$ do not diverge from each other (cf. proof of Lemma 4.4).

Step 1. $Y_{1}$ and $Y_{2}$ must centralize each other. Fix any $y_{0} \in Y_{2}$. Since $Y_{1}$ is a one-parameter subgroup, there is a surjective homomorphism $\rho: \mathbf{R} \rightarrow Y_{1}$. For 
any $t \in \mathbf{R}$, there is some $x \in Y_{2}$ within a bounded distance of $\rho(t)$; we write $\rho(t) \doteq x$. Because $Y_{2}$ is abelian and because $x \doteq \rho(t)$, we have

$$
y_{0}=x^{-1} y_{0} x \doteq \rho(t)^{-1} y_{0} \rho(t) .
$$

This means that the right-hand side of this equation is bounded (as a function of $t$ ). But (see Lemma 4.6) each entry of the matrix $\rho(t)^{-1}=\rho(-t)$ is a polynomial of exponentials; each matrix entry of $y_{0}$ is just a constant; and each matrix entry of $\rho(t)$ is a polynomial of exponentials. Multiplying these three matrices together results in a matrix whose entries are polynomials of exponentials. Because these entries are bounded, Lemma 4.5 asserts that these entries are constant. This means that $y_{0}=\rho(t)^{-1} y_{0} \rho(t)$ for all $t$; i.e., $y_{0}$ commutes with every element of $Y_{1}$. Because $y_{0}$ is an arbitrary element of $Y_{2}$, this implies that $Y_{1}$ and $Y_{2}$ commute.

Step 2. $Y_{1}$ and $Y_{2}$ are equal. Let $A$ be the closure of $Y_{1} Y_{2}$. Because step 1 asserts that $Y_{1}$ and $Y_{2}$ commute, it is obvious that $A$ is an abelian group. Because $Y_{2}$ does not diverge from $Y_{1}$ (and hence $A$ does not diverge from $Y_{1}$ ), it is easy to see that $A / Y_{1}$ is compact. Because $Y_{1}$ is simply connected, there is a compact subgroup $E$ of $A$ such that $A$ is the direct product $Y_{1} \times E$ (cf. [17, Exercise 37 of Chapter 3, p. 254]). This implies that $Y_{1}$ is the nonelliptic part of $Y_{2}$, and $E$ is the elliptic part of $Y_{2}$. Because $Y_{2}$ has no elliptic part, then $Y_{1}=Y_{2}$, as desired.

The extension of Theorem 4.7 to higher-dimensional subgroups requires the notion of elliptic part, and of nonelliptic part, for higher-dimensional subgroups.

(4.8) Definition. Let $X$ be a connected Lie subgroup of $\operatorname{GL}(n, \mathbf{R})$. The nonelliptic part of $X$ is the subgroup $Y$ of $\operatorname{GL}(n, \mathbf{R})$ generated by the collection of all the nonelliptic parts of the one-parameter subgroups of $X$. It turns out (see Proposition 3.9 and 3.10) that

(a) $Y$ is a closed, connected subgroup of $\operatorname{GL}(n, \mathbf{R})$;

(b) if $N$ is a connected, closed, normal Lie subgroup of $Y$ such that either $N$ or $Y / N$ is compact, then either $N$ is trivial or $N=Y$, respectively; and

(c) there is a compact, connected Lie subgroup $E$ of $\operatorname{GL}(n, \mathbf{R})$, such that $X E=Y E=\overline{X Y}$ (where $\overline{X Y}$ is the closure of $X Y$ ), and such that $E$ normalizes both $X$ and $Y$.

We will sometimes refer to the subgroup $E$ as the elliptic part of $X$; but this subgroup is often not unique.

(4.9) Theorem. Let $X$ and $Y$ be connected Lie subgroups of $G=G L_{n}(\mathbf{R})$. Assume $Y$ is unimodular. If $X$ does not diverge from $Y$, then the nonelliptic part of $X$ is contained in the nonelliptic part of $Y$.

Proof. Let $\mathscr{G}$ be the Lie algebra of $G=\mathrm{GL}(n \mathbf{R})$, let $\mathscr{Y}$ be the Lie algebra of $Y$ (so $\mathscr{Y} \subset \mathscr{G}$ ), and let $d=\operatorname{dim} Y$. Let $V=\bigwedge^{d} \mathscr{G}$ be the $d$ th exterior power of $\mathscr{G}$, and choose some nonzero element $v$ of the one-dimensional 
subspace $\wedge^{d} \mathscr{Y}$ of $V$. Now $G$ acts on $V$ by an exterior power of the adjoint representation; the stabilizer of $v$ in $G$ consists of those elements of $G$ that normalize $Y$, with modulus one. In particular, since $Y$ is unimodular, $Y$ fixes the vector $v$.

Because $Y$ fixes $v$, and $X$ does not diverge from $Y$, the orbit $v^{X}$ of $v$ under $X$ is precompact. We may assume that $X$ has no elliptic part. Then, since the representation of $G$ on $V$ is polynomial (i.e., the homomorphism $G \rightarrow \mathrm{GL}(V)$ is a morphism in the category of algebraic groups), this implies that $X$ stabilizes $v$ (see Lemmas 4.5 and 4.6). This means that $X$ must normalize $Y$.

We may assume that neither $X$ nor $Y$ has an elliptic part. Because $X$ normalizes $Y$, this implies that $\overline{X Y}$ is a subgroup that has no elliptic part. Now $X Y$ does not diverge from $Y$, so $\overline{X Y} / Y$ compact. Because $X Y$ has no elliptic part, then 4.8 (b) implies $\overline{X Y} / Y$ is trivial, i.e., $X$ is contained in $Y$, as desired.

4.10 Corollary. Let $X$ and $Y$ be connected Lie subgroups of a simply connected, nilpotent Lie group $N$. If $X$ does not diverge from $Y$, then $X \subset Y$.

Proof. Every subgroup of a nilpotent group is unimodular, and $N$ has a faithful representation as a unipotent group [14, $\S 1.4$ of the Preliminaries, p. 3], so there is no elliptic part.

(4.11) Corollary. Let $X$ and $Y$ be connected, unimodular Lie subgroups of a simply connected, solvable Lie group $G$. Assume every eigenvalue of every element of $\mathrm{Ad} G$ is real. If $X$ does not diverge from $Y$, then $X \subset Y$.

Proof. Because of the assumption on eigenvalues, $G$ has a faithful representation as a closed subgroup of the upper triangular matrices in $\operatorname{GL}(n, \mathbf{R})$ (see the proof of [17, Theorem 3.18.16, p. 246]). No subgroup of the upper triangular matrices has an elliptic part.

(4.12) Corollary. Let $X$ and $Y$ be connected Lie subgroups of $G=G L_{n}(\mathbf{R})$. Assume $Y$ is unimodular. If $X$ diverges from $Y$, then some one-parameter subgroup of $X$ diverges from $Y$.

Proof. If $X$ diverges from $Y$, then the nonelliptic part of $X$ cannot be contained in the nonelliptic part of $Y$; hence there is a one-parameter subgroup of $X$ whose nonelliptic part is not contained in the nonelliptic part of $Y$. The theorem asserts that this one-parameter subgroup diverges from $Y$.

(4.13) Corollary (of proof). Let $A$ and $Y$ be connected Lie subgroups of $G=$ $G L_{n}(\mathbf{R})$. Assume that $A$ is a one-parameter subgroup and that $Y$ is unimodular. If $A$ diverges from $Y$, then every doubly unbounded subset of $A$ diverges from $Y$.

Proof. Lemma 4.5 implies that any polynomial of exponentials is unbounded on any doubly unbounded subset of $\mathbf{R}$. 
Definition. The one-parameter subgroups of a Lie group $G$ are in natural oneto-one correspondence with the one-dimensional vector subspaces of the Lie algebra $\mathscr{G}$ of $G$. The collection of one-dimensional vector subspaces is simply the projective space associated to $\mathscr{G}$; this has a natural topology, and this topology defines a topology on the space of one-parameter subgroups.

(4.14) Corollary. Let $A$ and $Y$ be connected Lie subgroups of $G L_{n}(\mathbf{R})$. Assume that $A$ is a one-parameter subgroup and that $Y$ is unimodular. If $A$ diverges from $Y$, then every one-parameter subgroup sufficiently close to $A$ diverges from $Y$.

Proof. If the nonelliptic part of $Y$ does not contain the nonelliptic part of $A$, then it does not contain the nonelliptic part of any one-parameter subgroup sufficiently close to $A$.

(4.15) Corollary (of proof). Let $X$ and $Y$ be connected Lie subgroups of a real algebraic group $G$. Assume that $Y$ is a real algebraic subgroup and that the variety $Y \backslash G$ is affine. If $X$ does not diverge from $Y$, then the nonelliptic part of $X$ is contained in $Y$.

\section{TOPOLOGICAL RIGIDITY OF HOMOGENEOUS FOLIATIONS: THE SOLVABLE CASE}

(5.1) Theorem. For $i=1,2$, let $G_{i}$ be a simply connected, solvable Lie group; let $\Gamma_{i}$ be a lattice in $G_{i}$; and let $X_{i}$ be a connected Lie subgroup of $G_{i}$. Let $\mathscr{F}_{i}$ be the foliation of $\Gamma_{i} \backslash G_{i}$ by cosets of $X_{i}$. Assume

(a) $\mathscr{F}$ has a dense leaf; and

(b) $X_{i}$ is unimodular; and

(c) all the eigenvalues of every element of $\operatorname{Ad} G_{1}$ and of every element of $\operatorname{Ad} G_{2}$ are real.

If $f: \Gamma_{1} \backslash G_{1} \rightarrow \Gamma_{2} \backslash G_{2}$ is a homeomorphism that maps each leaf of $\mathscr{F}_{1}$ onto a leaf of $\mathscr{F}_{2}$, then $f$ is the composition of an affine map and a homeomorphism of $\Gamma_{2} \backslash G_{2}$ that maps each leaf of $\mathscr{F}_{2}$ onto itself.

Proof. This proof is mostly due to Benardete [3, §9].

Step 1. $f$ lifts to a homeomorphism $\tilde{f}: G_{1} \rightarrow G_{2}$. Because $G_{i}$ is the universal cover of $\Gamma_{i} \backslash G_{i}$, this is a consequence of elementary covering-space theory.

Step 2 (Benardete $[3, \S 8]$ ). We may assume $G_{1}=G_{2}, \Gamma_{1}=\Gamma_{2}$, and that the restriction of $\tilde{f}$ to $\Gamma_{1}$ is the identity. By composing each of $f$ and $\tilde{f}$ with a translation if necessary, we may assume that $f$ maps the basepoint $\Gamma_{1} e$ of $\Gamma_{1} \backslash G_{1}$ to the basepoint $\Gamma_{2} e$ of $\Gamma_{2} \backslash G_{2}$, and that $\tilde{f}$ maps the identity element of $G_{1}$ to the identity element of $G_{2}$. Then the restriction of $\tilde{f}$ to $\Gamma_{1}$ is an isomorphism of $\Gamma_{1}$ with $\Gamma_{2}$. The condition that the eigenvalues are real implies that this isomorphism (indeed, any isomorphism of $\Gamma_{1}$ with $\Gamma_{2}$ ) extends to an isomorphism of $G_{1}$ with $G_{2}$ (Theorem 2.2). If we identify $G_{1}$ with $G_{2}$ under this isomorphism, then we have the desired conclusions. 
Notation. In light of step 2, we will use the letter $G$ in the place of $G_{1}$ or $G_{2}$, and we will use the letter $\Gamma$ in the place of $\Gamma_{1}$ or $\Gamma_{2}$.

Step 3 (cf. Benardete $[3, \S 9]$ ). $X_{1}=X_{2}$. Lemma 2.3 asserts that $\Gamma \backslash G$ is compact (because $G$ is solvable). Then, because the restriction of $\tilde{f}$ to $\Gamma$ is the identity, the homeomorphism $\tilde{f}$ moves the points of $G$ by a bounded amount. Because $\tilde{f}$ maps the leaves of $\mathscr{F}_{1}$ to the leaves of $\mathscr{F}_{2}$, this implies that $X_{1}$ and $X_{2}$ do not diverge from each other. Therefore, Corollary 4.11 asserts that $X_{1}=X_{2}$.

Notation. In light of step 3, we will use the letter $X$ in the place of $X_{1}$ or $X_{2}$; we will use the letter $\mathscr{F}$ in the place of $\mathscr{F}_{1}$ or $\mathscr{F}_{2}$.

Step 4. For $g \in G$, define $\delta(g) \in G$ by: $\tilde{f}(g)=g \cdot \delta(g)$; then $\delta(g)$ normalizes $X$, i.e., $\delta(g) \in N_{G}(X)$. Because $\tilde{f}$ maps each $\mathscr{F}$-leaf to some $\mathscr{F}$-leaf, we have

$$
\tilde{f}(g X)=\tilde{f}(g) X=g \cdot \delta(g) X=g \cdot\left(\delta(g) X \delta(g)^{-1}\right) \cdot \delta(g) .
$$

As observed in step 3 , we know that $\tilde{f}$ moves the points of $G$ by only a bounded amount, so this equation implies that the subgroups $X$ and $\delta(g) X \delta(g)^{-1}$ do not diverge from each other; hence Corollary 4.11 implies that they are equal; this means that $\delta(g)$ normalizes $X$, as desired.

Step 5. $\tilde{f}(g X)=g X$ for every $g \in G$. Note that, because $\tilde{f}$ acts trivially on $\Gamma$, the map $\delta$ factors through to a map $\delta: \Gamma \backslash G \rightarrow G$. For $x \in X$, we have $\tilde{f}(g x)=\tilde{f}(g) \cdot x^{\prime}$ for some $x^{\prime} \in X$ (because $\tilde{f}$ maps leaves of $\mathscr{F}$ to leaves of $\mathscr{F})$, so

$$
g x \cdot \delta(g x)=\tilde{f}(g x)=\tilde{f}(g) x^{\prime}=g \cdot \delta(g) \cdot x^{\prime} .
$$

This implies that the quotient $\tilde{\delta}: \Gamma \backslash G \rightarrow N_{G}(X) / X$ is constant on the leaves of $\mathscr{F}$. Because $\mathscr{F}$ has a dense leaf, this implies that $\bar{\delta}$ is constant. Because $\tilde{f}$ has fixed points (it fixes each element of $\Gamma$ ), we know that $\delta(g)=e$ for some elements of $G$. Therefore, $\delta(g)$ belongs to $X$ for every $g \in G$, as desired.

(5.2) Corollary of proof (cf. Benardete [3, Theorem A]). For $i=1,2$, let $G_{i}$ be a simply connected solvable Lie group; let $\Gamma_{i}$ be a lattice in $G_{i}$; and let $X_{i}$ be a connected, unimodular Lie subgroup of $G_{i}$. Let $\mathscr{F}_{i}$ be the foliation of $\Gamma_{i} \backslash G_{i}$ by cosets of $X_{i}$. Assume either

(i) $G_{1}$ and $G_{2}$ are nilpotent, or

(ii) the eigenvalues of every element of $\operatorname{Ad} G_{1}$ and $\operatorname{Ad} G_{2}$ are real.

If there is a homeomorphism $f: \Gamma_{1} \backslash G_{1} \rightarrow \Gamma_{2} \backslash G_{2}$ that maps each leaf of $\mathscr{F}_{1}$ onto a leaf of $\mathscr{F}_{2}$, then there is an affine map $\phi: \Gamma_{1} \backslash G_{1} \rightarrow \Gamma_{2} \backslash G_{2}$ that maps each leaf of $\mathscr{F}_{1}$ onto a leaf of $\mathscr{F}_{2}$.

(5.3) Corollary. For $i=1,2$, let $G_{i}$ be a simply connected, nilpotent Lie group; let $\Gamma_{i}$ be a lattice in $G_{i}$; and let $X_{i}$ be a connected Lie subgroup of $G_{i}$. Let $\mathscr{F}_{i}$ be the foliation of $\Gamma_{i} \backslash G_{i}$ by cosets of $X_{i}$ and assume $\mathscr{F}_{i}$ has a dense leaf. If $f: \Gamma_{1} \backslash G_{1} \rightarrow \Gamma_{2} \backslash G_{2}$ is a homeomorphism that maps each leaf of $\mathscr{F}_{1}$ onto 
a leaf of $\mathscr{F}_{2}$, then $f$ is the composition of an affine map and a homeomorphism of $\Gamma_{2} \backslash \Gamma_{2}$ that maps each leaf of $\mathscr{F}_{2}$ onto itself.

Proof. Nilpotent groups are solvable. In a nilpotent group $G$, every subgroup is unimodular, and 1 is the only eigenvalue of any element of $\operatorname{Ad} G$.

\section{TOPOLOGICAL RIGIDITY OF HOMOGENEOUS FOLIATIONS: THE SEMISIMPLE CASE}

(6.1) Theorem. For $i=1,2$, let $G_{i}$ be a connected, semisimple Lie group with trivial center and no compact factor; let $\Gamma_{i}$ be a lattice in $G_{i}$; and let $X_{i}$ be a connected Lie subgroup of. $G_{i}$. Let $\mathscr{F}_{i}$ be the foliation of $\Gamma_{i} \backslash G_{i}$ by cosets of $X_{i}$. Assume

(a) $\mathscr{F}_{i}$ has a dense leaf; and

(b) $X_{i}$ is unimodular; and

(c) $G_{1}$ has no normal subgroup $N$, isomorphic to $\operatorname{PSL}(2, \mathbf{R})$, such that $N \Gamma_{1}$ is a closed subgroup of $G_{1}$.

If $f: \Gamma_{1} \backslash G_{1} \rightarrow \Gamma_{2} \backslash G_{2}$ is a homeomorphism that maps each leaf of $\mathscr{F}_{1}$ onto a leaf of $\mathscr{F}_{2}$, then $f$ is the composition of an affine map and a homeomorphism of $\Gamma_{2} \backslash G_{2}$ that maps each leaf of $\mathscr{F}_{2}$ onto itself.

Proof. This proof is based on Benardete's work [3, Theorem 5].

Step 1 [3, Lemma 1 of $\S 8$ ]. $f$ lifts to a homeomorphism $\tilde{f}: G_{1} \rightarrow G_{2}$. The theory of covering spaces implies that $f$ lifts to a homeomorphism $\hat{f}$ from the universal cover $\tilde{G}_{1}$ of $G_{1}$ to the universal cover $\tilde{G}_{2}$ of $G_{2}$. By composing $f$ with a translation if necessary, we may assume that $f$ maps the basepoint $\Gamma_{1} e$ of $\Gamma_{1} \backslash G_{1}$ to the basepoint $\Gamma_{2} e$ of $\Gamma_{2} \backslash G_{2}$; then $f$ induces an isomorphism $f_{*}$ of the fundamental group, $\pi_{1}\left(\Gamma_{1} \backslash G_{1}\right)$, of $\Gamma_{1} \backslash G_{1}$ with the fundamental group, $\pi_{1}\left(\Gamma_{2} \backslash G_{2}\right)$, of $\Gamma_{2} \backslash G_{2}$. Because $G_{i}$ is connected and has trivial center, one can see that $G_{i}=\widetilde{G}_{i} / Z\left(\pi_{1}\left(\Gamma_{i} \backslash G_{i}\right)\right)$. Because the isomorphism $f_{*}$ must map the center of $\pi_{1}\left(\Gamma_{1} \backslash G_{1}\right)$ to the center of $\pi_{1}\left(\Gamma_{2} \backslash G_{2}\right)$, it follows that $\hat{f}$ factors through to a map $\tilde{f}: G_{1} \rightarrow G_{2}$.

Step 2. We may assume $G_{1}=G_{2}, \Gamma_{1}=\Gamma_{2}$, and that the restriction of $\tilde{f}$ to $\Gamma_{1}$ is the identity. This is a consequence of the Mostow Rigidity Theorem (as extended by Prasad and Margulis; see Theorem 2.1). By composing each of $f$ and $\tilde{f}$ with a translation if necessary, we may assume that $f$ maps the basepoint $\Gamma_{1} e$ of $\Gamma_{1} \backslash G_{1}$ to the basepoint $\Gamma_{2} e$ of $\Gamma_{2} \backslash G_{2}$, and that $\tilde{f}$ maps the identity element of $G_{1}$ to the identity element of $G_{2}$. Then the restriction of $\tilde{f}$ to $\Gamma_{1}$ is an isomorphism of $\Gamma_{1}$ with $\Gamma_{2}$. The Mostow Rigidity Theorem 2.1 asserts that this isomorphism $\Gamma_{1} \rightarrow \Gamma_{2}$ extends to an isomorphism $\sigma: G_{1} \rightarrow G_{2}$. If we identify $G_{1}$ with $G_{2}$ under this isomorphism $\sigma$, then we have the desired conclusions.

Notation. In light of step 2, we will use the letter $G$ in the place of $G_{1}$ or $G_{2}$, and we will use the letter $\Gamma$ in the place of $\Gamma_{1}$ or $\Gamma_{2}$. 
Step 3. $X_{1}$ and $X_{2}$ have the same nonelliptic part; call it $Y$. For the moment, make the simplifying assumption that $\Gamma \backslash G$ is compact. Then, because the restriction of $\tilde{f}$ to $\Gamma$ is the identity, the homeomorphism $\tilde{f}$ moves the points of $G$ by a bounded amount. Because $\tilde{f}$ maps the leaves of $\mathscr{F}$ to the leaves of $\mathscr{F}_{2}$, this implies that $X_{1}$ and $X_{2}$ do not diverge from each other. Therefore, Theorem 4.9 implies that $X_{1}$ and $X_{2}$ have the same nonelliptic part, as desired.

Now consider the case where $\Gamma \backslash G$ is not compact. Suppose, for a contradiction, that $X_{1}$ diverges from $X_{2}$. Then Corollary 4.12 asserts that there is some one-parameter subgroup $A$ of $X_{1}$ that diverges from $X_{2}$.

Choose a compact subset $K$ of $\Gamma \backslash G$, such that $K$ has positive measure. Then $f$ is uniformly continuous on $K$; because $\tilde{f}$ restricts to the identity on $\Gamma$, this implies that $\tilde{f}$ moves the points of $\Gamma K$ only a bounded amount. The simple Poincaré Recurrence Theorem 2.7 (with $S=\Gamma \backslash G$ ) implies that, for almost any $g \in G$, the set $A_{g}=\{a \in A \mid g a \in \Gamma K\}$ is doubly unbounded in $A$.

Fix some elements $g \in G$, with $g$ very close to the identity element of $G$, such that $A_{g}$ is doubly unbounded in $A$. For any $a \in A_{g}$, we have $g a \in \Gamma K$, so $\tilde{f}(g a)$ is within a bounded distance of $g a$. Because $\tilde{f}$ maps the leaves of $\mathscr{F}_{1}$ to the leaves of $\mathscr{F}_{2}$ (and because $A \subset X_{1}$ ), we also know that $\tilde{f}(g a) \in \tilde{f}(g) \cdot X_{2}$. Hence $g \alpha$ is within a bounded distance of $\tilde{f}(g) \cdot X_{2}$. Because $\tilde{f}(e)=e$, and $g$ is very close to $e$, we must have $\tilde{f}(g)=g \varepsilon$, for some very small element $\varepsilon$ of $G$. Hence $g \alpha$ is within a bounded distance of $g \varepsilon \cdot X_{2}$, for every $a \in A_{g}$. This implies that $\varepsilon^{-1} A_{g} \varepsilon$ does not diverge from $X_{2}$.

Because the one-parameter subgroup $\varepsilon^{-1} A \varepsilon$ is very close to the one-parameter subgroup $A$, Corollary 4.14 asserts that $\varepsilon^{-1} A \varepsilon$ diverges from $X_{2}$. So Corollary 4.13 asserts that every doubly unbounded subset of $\varepsilon^{-1} A \varepsilon$ diverges from $X_{2}$. This contradicts the conclusion of the preceding paragraph.

Step 4. For $g \in G$, define $\delta(g) \in G$ by: $\tilde{f}(g)=g \cdot \delta(g)$; then $\delta(g)$ normalizes $Y$, i.e., $\delta(g) \in N_{G}(Y)$. Because $\tilde{f}$ maps $X_{1}$-leaves to $X_{2}$-leaves, we have

$$
\tilde{f}\left(g X_{1}\right)=\tilde{f}(g) X_{2}=g \cdot \delta(g) X_{2}=g \cdot\left(\delta(g) X_{2} \delta(g)^{-1}\right) \cdot \delta(g) .
$$

If $\Gamma \backslash G$ is compact, then $\tilde{f}$ moves points the points of $G$ by only a bounded amount, so this implies that the subgroups $X_{1}$ and $\delta(g) X_{2} \delta(g)^{-1}$ do not diverge from each other; hence Theorem 4.9 implies that they have the same nonelliptic part (namely $Y$ ); so it must be true that $\delta(g)$ normalizes $Y$.

We now consider the more difficult case where $\Gamma \backslash G$ is not compact. Let $g_{0}$ be an element of $G$ such that $Y \neq \delta\left(g_{0}\right) Y \delta\left(g_{0}\right)^{-1}$. Then $Y \not \subset \delta\left(g_{0}\right) Y \delta\left(g_{0}\right)^{-1}$, so Theorem 4.9 implies that $X_{1}$ diverges from $\delta\left(g_{0}\right) X_{2} \delta\left(g_{0}\right)^{-1}$; hence Corollary 4.12 asserts that there is some one-parameter subgroup $A$ of $X_{1}$ that diverges from $\delta\left(g_{0}\right) X_{2} \delta\left(g_{0}\right)^{-1}$.

Let $K$ and $A_{g}$ be as in the proof of step 3. The Poincare Recurrence Theorem 2.7 implies that we can choose some $g \in G$, very close to $g_{0}$, such that 
$A_{g}$ is doubly unbounded in $A$. Corollary 4.14 implies that $A$ diverges from $\delta(g) X_{2} \delta(g)^{-1}$, so Corollary 4.13 asserts that $A_{g}$ diverges from $\delta(g) X_{2} \delta(g)^{-1}$.

On the other hand, we know that

$$
\tilde{f}\left(g X_{1}\right)=g \cdot\left(\delta(g) X_{2} \delta(g)^{-1}\right) \cdot \delta(g) .
$$

Because $A_{g} \subset X_{1}$, and because $\tilde{f}$ moves points of $\Gamma K$ by only a bounded amount, this implies that $A_{g}$ does not diverge from $\delta(g) X_{2} \delta(g)^{-1}$. This contradicts the conclusion of the preceding paragraph.

Step 5. Let $E_{1}$ and $E_{2}$ be the elliptic parts of $X_{1}$ and $X_{2}$; then $\delta(g)$ belongs to $E_{1} E_{2} Y$ for every $g \in G$. Note that, because $\tilde{f}$ acts trivially on $\Gamma$, the map $\delta$ factors through to a map $\delta: \Gamma \backslash G \rightarrow G$. For $x_{1} \in X_{1}$, we have $\tilde{f}\left(g x_{1}\right)=\tilde{f}(g) \cdot x_{2}$ for some $x_{2} \in X_{2}$, so

$$
g x_{1} \cdot \delta\left(g x_{1}\right)=\tilde{f}\left(g x_{1}\right)=\tilde{f}(g) x_{2}=g \cdot \delta(g) \cdot x_{2} .
$$

Writing $x_{1}=e_{1} y_{1}$ and $x_{2}=e_{2} y_{2}$ for some $e_{1} \in E_{1}, e_{2} \in E_{2}$, and $y_{1}, y_{2} \in$ $Y$, we then have $e_{1} y_{1} \cdot \delta\left(g x_{1}\right)=\delta(g) \cdot e_{2} y_{2}$. This implies that the quotient $\bar{\delta}: \Gamma \backslash G \rightarrow E_{1} \backslash N_{G}(Y) / Y E_{2}$ is constant on the leaves of $\mathscr{F}_{1}$. Because $\mathscr{F}_{1}$ has a dense leaf, this implies that $\bar{\delta}$ is constant. Because $\tilde{f}$ has fixed points (it fixes each element of $\Gamma$ ), we know that $\delta(g)=e$ for some elements of $G$. Therefore, $\delta(g)$ belongs to $E_{1} E_{2} Y$ for every $g \in G$, as desired.

Remark. If $X_{1}$ and $X_{2}$ have no elliptic part (so $X_{1}=X_{2}=Y$ and $E_{1}=$ $E_{2}=e$, and $\left.\mathscr{F}_{1}=\mathscr{F}_{2}\right)$, the conclusion of step 5 implies that $\tilde{f}$ maps each $\mathscr{F}_{2}$-leaf onto itself, which completes the proof of the theorem. In the general case, we need to continue the argument in order to conclude that $X_{1}=X_{2}$ and that $\tilde{f}$ maps each $\mathscr{F}_{2}$-leaf onto itself. The idea is that, because $X_{1}$ and $X_{2}$ differ by only a compact amount, the results we want are true, modulo an equivariant map into a compact subgroup. Because the action of $X_{1}$ on $\Gamma \backslash G$ is weak-mixing, Lemma 2.8 will imply that any equivariant map into a compact subgroup is trivial.

Step 6. $X_{1}$ and $X_{2}$ have the same elliptic part; call it $E$. Let $x \in X_{1}$. We have

$$
g x \cdot \delta(g x)=\tilde{f}(g x) \in \tilde{f}\left(g X_{1}\right)=\tilde{f}(g) \cdot X_{2} \subset \tilde{f}(g) \cdot Y E_{2}=g \cdot \delta(g) \cdot Y E_{2} .
$$

Therefore $x \cdot \delta(g x) \in \delta(g) \cdot Y E_{2}$; writing $x=e y$, with $e \in E_{1}$ and $y \in Y$ (and using the fact that $E_{1}$ and $E_{2}$ normalize $Y$, because the elliptic part of a group always normalizes the nonelliptic part), we find that $e \cdot \delta(g x) \in \delta(g) \cdot Y E_{2}$. Now $\delta$ induces a map

$$
\hat{\delta}: \Gamma \backslash G \rightarrow E_{1} E_{2} Y / E_{2} Y \cong E_{1} /\left(E_{1} \cap E_{2} Y\right)
$$

into $E_{1} /\left(E_{1} \cap E_{2} Y\right)$. We find that $e \cdot \hat{\delta}(g x)=\hat{\delta}(g)$. Now Lemma 2.8(b) asserts that the set

$$
\widehat{E}=\left\{e \in E_{1} \mid e y \in X_{1} \text { for some } y \in Y\right\}
$$


is contained in an $E_{1}$-conjugate of $E_{1} \cap E_{2} Y$. Because Proposition 3.10(e) asserts that $\widehat{E}$ is dense in $E_{1}$, we conclude that $E_{1} \subset E_{2} Y$. By symmetry, we must have $E_{1} Y=E_{2} Y$, i.e., that $X_{1}$ and $X_{2}$ have the same elliptic part.

Step 7. $\tilde{f}(g) \in g \cdot X_{2}$ for all $g \in G$. Because $E=E_{1}=E_{2}$, step 5 asserts that $\delta(g) \in E Y$ for all $g \in G$. Lemma 3.9(iv) asserts that $E Y=E X_{2}$, so $\delta$ determines a well-defined map $\delta^{\prime}: \Gamma \backslash G \rightarrow E /\left(E \cap X_{2}\right)$, defined by: $\delta(g) \in \delta^{\prime}(g) \cdot X_{2}$.

For $x \in X_{1}$, we have

$$
\begin{aligned}
g x \cdot \delta^{\prime}(g x) \cdot X_{2} & =g x \cdot \delta(g x) \cdot X_{2} \\
& =\tilde{f}(g x) \cdot X_{2}=\tilde{f}(g) \cdot X_{2}=g \cdot \delta(g) \cdot X_{2}=g \cdot \delta^{\prime}(g) \cdot X_{2} .
\end{aligned}
$$

Therefore, writing $x=e y$ with $e \in E$ and $y \in Y$, and using the fact that $E$ commutes with $Y$, modulo $X_{2} \cap Y$ (see Lemma 3.10d), we have

$$
e \cdot \delta^{\prime}(g x) y \cdot X_{2}=x \cdot \delta^{\prime}(g x) \cdot X_{2}=\delta^{\prime}(g) \cdot X_{2} .
$$

Now choose $d \in E$ such that $d y \in X_{2}$. (Note that $d$ is well defined, modulo $E \cap X_{2}$.) Using the fact that $E$ (in particular, that $d$ ) normalizes $E \cap X_{2}$ (because it normalizes both $E$ and $X_{2}$ ), we find that

$$
e d^{-1} \cdot \delta^{\prime}(g x)=\delta^{\prime}(g) \text {. }
$$

Now Lemma 2.8 implies that $\delta^{\prime}$ is constant. Since $\tilde{f}$ has fixed points, we conclude that $\delta^{\prime}=e$; hence $\tilde{f}(g) \in g \cdot X_{2}$ for all $g \in G$.

Step 8. For any $g \in G, \tilde{f}$ maps the $\mathscr{F}_{2}$-leaf through $g$ onto itself. For $x \in X_{1}$, step 7 implies that $\tilde{f}(g x) \in g x \cdot X_{2}$. Because $\tilde{f}$ maps $\mathscr{F}_{1}$-leaves onto $\mathscr{F}_{2}$-leaves, and because $x \in X_{1}$, it follows that

$$
g \cdot X_{2}=\tilde{f}\left(g \cdot X_{1}\right)=\tilde{f}\left(g x \cdot X_{1}\right)=g x \cdot X_{2} .
$$

Therefore, $x \in X_{2}$. Because $x$ is an arbitrary element of $X_{1}$, this implies $X_{1} \subset X_{2}$. By symmetry, we must have $X_{1}=X_{2}$; hence $\mathscr{F}_{1}$ and $\mathscr{F}_{2}$ are the same foliation. But step 7 implies that $\tilde{f}$ maps the $\mathscr{F}_{1}$-leaf through $g$ onto the $\mathscr{F}_{2}$-leaf through $g$, so we conclude, as desired, that $\tilde{f}$ maps the $\mathscr{F}_{2}$-leaf through $g$ onto itself.

\section{Complements}

In this section, we consider the consequence of eliminating hypothesis (a), (b), or (c) from Theorem 1.1. Without hypothesis (a), one expects there to be many topological equivalences of $\mathscr{F}_{i}$ with itself, and, generally, very few of them will be of the elementary form described in the theorem. (If hypothesis (a) is omitted from Theorem 1.1, and case (iii) of hypothesis (c) is not allowed, then Corollary 5.2 shows that if there is a topological equivalence, then some topological equivalence has the elementary form described in the theorem. For one-dimensional foliations, this is due to Benardete.) 
Condition (b) is included in Theorem 1.1 to guarantee that the subgroups under consideration have good divergence properties (see Theorem 4.9). This condition may not be necessary in the theorem, but something like it seems to be necessary for Benardete's method to be applicable. For example, let $G$ be a compact, simply connected group. No two subgroups of $G$ diverge from each other, so Benardete's method does not seem to apply to foliations of $G$ by cosets of a subgroup. For another kind of example, let $P$ be a parabolic subgroup of any connected Lie group $G$; then $G / P$ is compact, so no subgroup of $G$ diverges from $P$, so Benardete's method will not be useful in distinguishing between foliations by cosets of various parabolic subgroups.

Remark. Let $P$ and $Q$ be parabolic subgroups of a noncompact, simple Lie group $G$ with trivial center; and let $\Gamma$ be a lattice in $G$. Amos Nevo (oral communication) pointed out that if the foliation of $\Gamma \backslash G$ by cosets of $P$ is topologically equivalent to the foliation by cosets of $Q$, then the action of $P$ on $\Gamma / G$ is orbit-equivalent to the action of $Q$. A parabolic subgroup is amenable if and only if it is minimal, so the fact that amenability is an invariant of orbitequivalence [18, Corollary 4.3.11, p. 82] implies that if $P$ is a minimal parabolic subgroup (hence amenable), then $Q$ is also a minimal parabolic subgroup; so $P$ and $Q$ are conjugate. Furthermore, the Zimmer super-rigidity theorem for cocycles implies (see [18, Theorem 5.2.14, pp. 111-112]) that $P / \operatorname{Amen}(P)$ is locally isomorphic to $Q / \operatorname{Amen}(Q)$ if $\mathbf{R}-\operatorname{rank}(P / \operatorname{Amen}(P)) \geq 2$, where $\operatorname{Amen}(X)$ is the maximal connected, closed, normal, amenable subgroup of $X$.

Condition (c) cannot be omitted from Theorem 1.1. For example, it has long been known that there are foliations of homogeneous spaces of nonabelian solvable groups that are topologically identical to foliations of abelian groups (see [3, Example 4 of $\S 10]$ ). It is also well known that the theorem fails for $G_{1}=G_{2}=\operatorname{PSL}(2, \mathbf{R})$ (see Theorem 7.3). It would be very interesting to know for which groups $G_{i}$ and which subgroups $X_{i}$ the theorem fails.

(7.1) Remark. Every connected, proper Lie subgroup of $\operatorname{SL}(2, \mathbf{R})$ is conjugate to one of the following:

(a) Compact:

$$
K=\left\{\left(\begin{array}{cc}
\cos \theta & \sin \theta \\
-\sin \theta & \cos \theta
\end{array}\right) \mid \theta \in \mathbf{R}\right\}
$$

(b) Diagonal:

$$
A=\left\{\left(\begin{array}{cc}
a & 0 \\
0 & a^{-1}
\end{array}\right) \mid a \in \mathbf{R}^{+}\right\}
$$

(c) Borel:

$$
B=\left\{\left(\begin{array}{cc}
a & x \\
0 & a^{-1}
\end{array}\right) \mid a \in \mathbf{R}^{+}, x \in \mathbf{R}\right\}
$$

or

(d) Unipotent:

$$
U=\left\{\left(\begin{array}{cc}
1 & x \\
0 & 1
\end{array}\right) \mid x \in \mathbf{R}\right\}
$$


As discussed in [15, §2], we can identify $\Gamma \backslash G / K$ with a compact surface $M$ of constant negative curvature, with fundamental group $\Gamma$; and $\Gamma \backslash G$ can be identified with the unit tangent bundle, $T^{1} M$, of $M$. This results in geometric interpretations of the foliations of $\Gamma \backslash G$ by cosets of $K, A, B$, or $U$ : the foliation of $\Gamma \backslash G$ by cosets of $K$ is simply the foliation of $T^{1} M$ by fibers of the canonical projection $T^{1} M \rightarrow M$; the foliation of $\Gamma \backslash G$ by cosets of $A$ is the foliation of $T^{1} M$ by orbits of the geodesic flow; the foliation of $\Gamma \backslash G$ by cosets of $B$ is the weak-stable foliation of the geodesic flow on $M$; the foliation of $\Gamma \backslash G$ by cosets of $U$ is the foliation of $T^{1} M$ by horocycles.

The following lemma is an algebraic formulation of the well-known geometric fact that if two compact surfaces of constant negative curvature have the same fundamental group, then they are homeomorphic but need not be isometric.

(7.2) Lemma (moduli space is nontrivial). Let $\Gamma$ be a cocompact lattice in $G=\operatorname{PSL}(2, \mathbf{R})$. There are (many!) lattices $\Gamma^{\prime}$ in $G$ such that $\Gamma \backslash G$ and $\Gamma^{\prime} \backslash G$ are homeomorphic, but such that $\Gamma^{\prime}$ and $\Gamma$ are not conjugate under Aut $G$.

The following well-known, classical result shows that Theorem 1.1 is not true for foliations by cosets of the subgroups $A, K$, and $B$ of $\operatorname{PSL}(2, \mathbf{R})$. Theorem 7.4 shows (for cocompact lattices) that Theorem 1.1 is true for foliations by cosets of the other subgroup, $U$. In Theorem 7.4, the fact that some topological equivalence of $\mathscr{F}_{1}$ and $\mathscr{F}_{2}$ is an affine map was proved by B. Marcus; that all topological equivalences are of a simple form was later proved by Ted Jones.

(7.3) Theorem (Folklore). In the notation of Remark 7.1, let $X$ be either the subgroup $A, K$, or $B$ of $G=P S L(2, \mathbf{R})$. Let $\Gamma$ and $\Gamma$ be any two torsion-free, cocompact lattices in $G$, such that $\Gamma \backslash G$ and $\Gamma^{\prime} \backslash G$ are homeomorphic. Then the foliation of $\Gamma \backslash G$ by cosets of $X$ is topologically equivalent to the foliation of $\Gamma^{\prime} \backslash G$ by cosets of $X$. (But, if $\Gamma$ and $\Gamma^{\prime}$ are not conjugate under $\operatorname{Aut}(G)$, there is no affine map from $\Gamma \backslash G$ onto $\Gamma^{\prime} \backslash G$.)

Idea of proof (cf. [1, pp. 25-26]). Suppose $M$ and $M^{\prime}$ are two compact surfaces of constant negative curvature, whose fundamental groups are isomorphic. (a) Then $M$ and $M^{\prime}$ are diffeomorphic; any diffeomorphism lifts to a homeomorphism of $T^{1} M$ with $T^{1} M^{1}$ that is a topological equivalence of the foliations by fibers of the projection to the surface.

(b) A diffeomorphism of $M$ with $M^{\prime}$ lifts to a diffeomorphism of the universal cover of $M$ with the universal cover of $M^{\prime}$. We can take the universal cover of $M$ (and $M^{\prime}$ ) to be the open unit disk $D$. Then the metric of constant negative curvature of $M$ (resp., on $M^{\prime}$ ) defines a Riemannian metric of constant negative curvature $\sigma$ (resp., $\sigma^{\prime}$ ) on $D$. Both $\sigma$ and $\sigma^{\prime}$ are $\Gamma$-invariant.

Oriented geodesics on $D$ (in either metric) are in one-to-one correspondence with ordered pairs of points on the boundary of $D$ : a geodesic corresponds to its two endpoints. It follows that, for each $\sigma$-geodesic, there is a unique $\sigma^{\prime}$-geodesic that has the same endpoints on the boundary. There is a homeomorphism $h$ of $D$ that takes each $\sigma$-geodesic onto the $\sigma^{\prime}$-geodesic with the 
same endpoints; $h$ can be chosen so that it commutes with the action of $\Gamma$. Then $h$ factors through to a homeomorphism of $\Gamma \backslash D$ that maps $M$-geodesics onto $M^{\prime}$-geodesics.

(c) Leaves of the weak-stable foliation of $D$ are in one-to-one correspondence with points on the boundary of $D$ : a leaf of the weak-stable foliation consists of those geodesics with a given endpoint at $+\infty$. By construction, the homeomorphism $h$ of the preceding paragraph maps $\sigma$-geodesics with a given endpoint at $+\infty$ to $\sigma^{\prime}$-geodesics with the same endpoint at $+\infty$. Hence, $h$ maps weak-stable leaves of the geodesic flow for $\sigma$ to weak-stable leaves of the geodesic flow for $\sigma^{\prime}$.

(7.4) Theorem (Marcus [10], T. Jones (oral communication)). Let $\Gamma_{1}$ and $\Gamma_{2}$ be cocompact lattices in $G=P S L(2, \mathbf{R})$, and let $U$ be the unipotent subgroup of $G$ as in Remark 7.1. Let $\mathscr{F}_{i}$ be the foliation of $\Gamma_{i} \backslash G$ by cosets of $U$. If $f: \Gamma_{1} \backslash G \rightarrow \Gamma_{2} \backslash G$ is a homeomorphism that maps each leaf of $\mathscr{F}_{1}$ onto a leaf of $\mathscr{F}_{2}$, then $f$ is the composition of an affine map and a homeomorphism of $\Gamma_{2} \backslash G$ that maps each leaf of $\mathscr{F}_{2}$ onto itself.

\section{Foliations of double-COSET SPACES}

The geodesic flow on the unit tangent bundle of a compact manifold of constant negative curvature is a flow, not on a homogeneous space, but on a double-coset space $\Gamma \backslash G / M$. Such examples are the reason that results, such as the following, on foliations of double-coset spaces are of interest.

(8.1) Proposition. For $i=1,2$, let $G_{i}$ be a connected, semisimple Lie group with trivial center and no compact factor, connected subgroup of $G_{i}$ that normalizes $X_{i}$. The foliation of $\Gamma_{i} \backslash G_{i}$ by cosets of $X_{i}$ projects to a foliation $\mathscr{F}_{i}$ on $\Gamma_{i} \backslash G_{i} / M_{i}$. Assume

(a) $\mathscr{F}_{i}$ has a dense leaf; and

(b) $X_{i}$ is unimodular; and

(c) $G_{1}$ has no normal subgroup $N$ isomorphic to $P S L(2, \mathbf{R})$, such that $N \Gamma_{1}$ is closed; and

(d) $X_{i}$ has no elliptic part.

If $f: \Gamma_{1} \backslash G_{1} / M_{1} \rightarrow \Gamma_{2} \backslash G_{2} / M_{2}$ is a homeomorphism that maps each leaf of $\mathscr{F}_{1}$ onto a leaf of $\mathscr{F}_{2}$, then $f$ is the composition of an affine map and a homeomorphism of $\Gamma_{2} \backslash G_{2} / M_{2}$ that maps each leaf of $\mathscr{F}_{1}$ onto itself.

Proof. Much the same as Theorem 6.1; we give only an outline of the necessary steps.

Step 1. $f$ lifts to a homeomorphism $\tilde{f}: G_{1} / M_{1} \rightarrow G_{2} / M_{2}$. Because $\Gamma_{i}$ is torsion-free, $G_{i} / M_{i}$ is a covering space of $\Gamma_{i} \backslash G_{i} / M_{i}$.

Step 2. We may assume $G_{1}=G_{2}, \Gamma_{1}=\Gamma_{2}, M_{1}=M_{2}$, and that the restriction of $\tilde{f}$ to $\Gamma_{1} M_{1} / M_{1}$ is the identity. Apply the Mostow Rigidity Theorem. 
Step 3. $X_{1}=X_{2}$. Because $X_{1}$ and $X_{2}$ have no elliptic part, it suffices to show that $X_{1} M_{1}$ and $X_{2} M_{2}$ have the same nonelliptic part, i.e., that they do not diverge from each other.

Step 4. For $g \in G$, define $\delta(g) \in G / M_{2}$ by: $\tilde{f}\left(g M_{1}\right)=g \cdot \delta(g)$; then $\delta(g) \in$ $N_{G}\left(X_{2}\right) / M_{2}$.

Step 5. $\delta(g) \in X_{2} M_{2} / M_{2}$ for every $g \in G$.

\section{REFERENCES}

1. D. V. Anosov (translated by S. Feder), Geodesic flows on closed Riemann manifolds with negative curvature (Proc. Steklov Inst. Math., no. 90), Amer. Math. Soc., Providence, R.I., 1969.

2. W. Baily, Introductory lectures on automorphic forms, Princeton University Press, Princeton, N.J., 1973.

3. D. Benardete, Topological equivalence of flows on homogeneous spaces, and divergence of oneparameter subgroups of Lie groups, Trans. Amer. Math. Soc. 306 (1988), 499-527.

4. I. P. Cornfeld, S. V. Fomin, and Y. G. Sinai, Ergodic theory, Springer-Verlag, New York, 1982.

5. V. V. Gorbacevič, Lattices in solvable Lie groups and deformations of homogeneous spaces, Math. USSR-Sb. 20 (1973), 249-266=Mat. Sb. (N.S.) 91(133) (1973), 233-252, 288.

6. S. Helgason, Differential geometry, Lie groups, and symmetric spaces, Academic Press, New York, 1978.

7. M. Hirsch and S. Smale, Differential equations, dynamical systems, and linear algebra, Academic Press, New York, 1974.

8. G. P. Hochschild, Basic theory of algebraic groups and Lie algebras, Springer-Verlag, New York, 1981.

9. J. E. Humphreys, Linear algebraic groups, Springer-Verlag, New York, 1975.

10. B. Marcus, Topological conjugacy of horocycle flows, Amer. J. Math. 105 (1983), 623-632.

11. C. C. Moore, Ergodicity of flows on homogeneous spaces, Amer. J. Math. 88 (1966), 154-178.

12. G. D. Mostow, Strong rigidity of locally symmetric spaces, Ann. of Math. Studies, no. 78, Princeton Univ. Press, Princeton, N.J., 1973.

13. G. Prasad, Strong rigidity of Q-rank 1 lattices, Invent. Math. 21 (1973), 255-286.

14. M. S. Raghunathan, Discrete subgroups of Lie groups, Springer-Verlag, New York, 1972.

15. M. Ratner, Ergodic theory in hyperbolic space, Conference in Modern Analysis and Probability (New Haven, Conn., 1982), Contemp. Math., vol. 26 (R. Beals, A. Beck, A. Bellow, and A. Hajian, eds.), Amer. Math. Soc., Providence, R.I., 1984, pp. 309-334.

16. M. Saito, Sur certains groupes de Lie résolubles II, Sci. Papers Coll. Gen. Ed. Univ. Tokyo 7 (1957), 157-168.

17. V. S. Varadarajan, Lie groups, Lie algebras, and their representations, Prentice-Hall, Englewood Cliffs, N.J., 1974.

18. R. J. Zimmer, Ergodic theory and semisimple groups, Birkhäuser, Boston, Mass., 1984.

Mathematical Sciences Research Institute, 1000 Centennial Drive, Berkeley, CaliFORNIA 94720

Current address: Department of Mathematics, Arizona State University, Tempe, Arizona 85287 